\title{
Proteomic analysis exploring the mechanism of bladder fibrosis induced by ketamine using a rat model
}

\author{
Quan Zhu ${ }^{1,2}$, Haozhen $\mathrm{Li}^{1,2}$, Kaixuan $\mathrm{Li}^{1,2}$, Zhao Wang ${ }^{1,2}$, Zhengyan Tang ${ }^{1,2}$ \\ ${ }^{1}$ Department of Urology, Xiangya Hospital, Central South University, Changsha, China; ${ }^{2}$ Provincial Laboratory for Diagnosis and Treatment of \\ Genitourinary System Disease, Changsha, China \\ Contributions: (I) Conception and design: Z Wang, ZY Tang; (II) Administrative support: Z Wang, ZY Tang; (III) Provision of study materials: Z \\ Wang, ZY Tang; (IV) Collection and assembly of data: Q Zhu, HZ Li, KX Li; (V) Data analysis and interpretation: Q Zhu; (VI) Manuscript writing: \\ All authors; (VII) Final approval of manuscript: All authors. \\ Correspondence to: Zhengyan Tang, Zhao Wang. Department of Urology, Xiangya Hospital, Central South University; 87 Xiangya Road, Changsha, \\ 410008, China. Email: xytzyan@163.com; xywangz07@163.com.
}

Background: Long-term abuse of ketamine can cause irreversible bladder fibrosis, but the mechanism of bladder fibrosis is still under investigation. This study aims to explore the mechanism of bladder fibrosis utilizing proteomic analysis in a rat model.

Methods: After building a rat model, hematoxylin and eosin (HE) staining, Masson's trichrome staining, and western blotting $(\mathrm{WB})$ of collagen I were used to assess bladder pathology and fibrosis in a rat model. Next, protein expression changes in the rat bladder by proteomic technology were quantitatively detected, and reverse transcription-polymerase chain reaction (RT-PCR) and WB were used to verify the expression of proteins. Bioinformatic techniques and functional analysis were also performed.

Results: Compared to the control group, thinning of the bladder epithelium layer, infiltration of submucosal inflammatory cells, deposition of many collagen fibers, and an elevated expression of collagen I were observed in the experimental group. A total of 3,690 proteins were identified, of which 423 proteins were upregulated, and 304 proteins were down-regulated. Eight out of ten mRNA expressions and acyloxyacyl hydrolase (AOAH), mast cell (MC) protease 1 protein expressions were in line with the proteomic results. Sixty-five differential expression proteins (DEPs) were found to belong to the immune system, and 18 of them were involved in immune diseases, according to KEGG analysis.

Conclusions: We consider that MC protease 1 and platelet activation pathways may play an important role in ketamine-induced bladder fibrosis.

Keywords: Proteomic; bladder fibrosis; ketamine

Submitted Apr 23, 2021. Accepted for publication Jul 02, 2021.

doi: $10.21037 /$ tau-21-351

View this article at: https://dx.doi.org/10.21037/tau-21-351

\section{Introduction}

Ketamine is a derivative of phencyclidine. It has been utilized as an analgesic and sedative in clinical settings since the 1960s (1). It is a new type of drug with popularity amongst the youth due to its strong hallucinogenic effects and low price (1). Recently, epidemiological studies suggest that ketamine abuse has become a serious social problem in Central and South Asia (2).
In 2007, Shahani et al. first reported that some people who had been illegally abusing ketamine had obvious urinary tract symptoms such as frequent urination, urgency, hematuria, and dysuria. After long-term abuse, bladder fibrosis developed, and the bladder's capacity was reduced. This series of changes are known as ketamine-associated urinary system dysfunction (KAUD) (3). Subsequent clinical studies found that some patients with ketamine cystitis (KC) are sometimes also affected by vesicoureteral reflux, 
bilateral upper urinary tract distention, and hydronephrosis, and some even with acute or chronic renal insufficiency, which seriously affects the patient's survival and quality of life (4).

Song et al. successfully established a model of ketamineassociated bladder fibrosis in rats by continuous intravenous injection of ketamine for two weeks. The study found that submucosal bladder apoptosis cells increased with the concentration of ketamine administered, and it was thought that apoptosis might play an important role in the pathogenesis of bladder fibrosis (5). Another study revealed that TGF- $\beta 1$ might be an important factor in the pathogenesis of ketamine-induced bladder fibrosis by regulating epithelial-mesenchymal transition (EMT) (6). Some experiments have been conducted to investigate ketamine-induced bladder fibrosis, but the mechanisms are still unclear. Therefore, revealing the mechanisms of bladder fibrosis is a challenging undertaking.

It is a new approach to study the mechanisms of bladder fibrosis induced by ketamine using proteomics. Proteomics is considered a key step in relay genomics and transcriptomics of biological research. Commonly used methods of proteomic research are two-dimensional gel electrophoresis, MALDI-TOF/MS, and SILAC (7). Ross et al. first proposed the isobaric tag for relative and absolute quantitation (iTRAQ) technology in 2004. iTRAQ is more sensitive and accurate than traditional proteomics research methods. A large number of researchers use this technology for protein quantitative analytic studies (8). Some researchers have analyzed the plasma proteins of patients with idiopathic pulmonary fibrosis and healthy controls by iTRAQ technology, and five proteins with different expressions were found (9). In the study of liver fibrosis, Cao et al. used iTRAQ combined with the liquid chromatography-tandem mass spectrometry method to find a total of 115 differently expressed proteins using a combination of a therapy group and the $\mathrm{CCl} 4$ induced liver fibrosis group in a rat model. Finally, the Wen study explained the mechanism of combination therapy against liver fibrosis and considered it a promising treatment (10).

The fact that ketamine causes bladder damage is widely recognized, but not much is known about which molecules play a role in developing bladder fibrosis. This study hypothesizes that immune factors may play an important role in the process of ketamine-induced bladder fibrosis, and it establishes a model for the inducement of bladder fibrosis by injection of ketamine via the tail vein of Sprague Dawley (SD) rats for 8 weeks. HE staining, Masson's trichrome staining, and western blotting (WB) of collagen I were used to assess bladder pathology and confirm the occurrence of bladder fibrosis. iTRAQ was then combined with LC-MS/MS technology to study the differences in protein expression between ketamine-treated rats and normal controls to explore the possible mechanisms of bladder fibrosis further. Reverse transcription-polymerase chain reaction PCR (RT-PCR) and WB were used to verify protein expression. Bioinformatic techniques and functional analysis of differential expression proteins (DEPs) were performed to find potentially related molecules and pathways. We present the following article in accordance with the ARRIVE Checklist (available at https://dx.doi. org/10.21037/tau-21-351).

\section{Methods}

\section{Animal feeding and injection of ketamine hydrochloride}

The experimental animals were female Sprague Dawley (SD) rats (200-250 g, SPF) purchased from Hunan Slack Jingda Experimental Animal Corporation (Hunan, China). Experiments were performed under a project license (NO.: 2018sydw0225) granted by Experimental Animal Welfare from Central South University, in compliance with Chinese guidelines for the care and use of animals. Animals were raised at the Experimental Animal Center of Central South University (Hunan, China). There were no more than 3 rats per cage. Temperature: $20-26^{\circ} \mathrm{C}$, relative humidity: $40-70 \%$, 12 hours of alternating light and dark cycles, regular feeding, replacement of bedding every 3-4 days, and the animals were also subjected to environmental adaptation for 1 week before the experiment. Sixteen SD rats were randomly divided into 2 groups; 8 rats in the experimental group and 8 rats in the control group, with the following treatment; (I) control group: tail vein injection of ketamine saline, (II) experimental group: tail vein injection of ketamine hydrochloride solution, $25 \mathrm{mg} / \mathrm{kg} /$ day $(2 \mathrm{~mL} / 0.1 \mathrm{~g}$, Fujian Gutian Pharmaceutical Corporation) for 8 weeks. Each rat was weighed weekly to adjust the required amount of ketamine hydrochloride solution. After 8 weeks, the rats were sacrificed with $2 \%$ pentobarbital, and the bladder of each rat was removed and divided into 3 portions for histological observation, $\mathrm{WB}$, and protein mass spectrometry.

\section{Histopathological observation}

The removed bladder tissue was fixed in $4 \%$ 
paraformaldehyde, and after 24 hours, was embedded into a wax block to prepare a $4 \mu \mathrm{m}$ section for use in subsequent observations. Hematoxylin and eosin staining was used to observe the morphology and inflammatory cell infiltration of the bladder tissue, and the distribution of collagen was observed by using Masson's trichrome staining.

\section{iTRAQ labeling and fractionation by high-PH reverse chromatography}

The vacuum-dried trypsin-digested peptides were labeled with an 8-plex iTRAQ (Sciex, USA) reagent, and the reaction was stopped by the addition of double-distilled $\mathrm{H}_{2} \mathrm{O}$ for 30 minutes. All labeled samples were then placed in a test tube, centrifuged, and dissolved in $110 \mu \mathrm{L}$ of mobile phase $\mathrm{A}(10 \mathrm{mM}$ ammonium formate, $5 \%$ aqueous acetonitrile, $\mathrm{pH}, 10.0$ ) in a high $\mathrm{pH}$ reverse column (Agilent, ZORBAX). One-dimensional fractionation was performed on a high-pH reverse column (Agilent, ZORBAX Extended-C18 2.1) using a separation gradient of buffer B (10 mM ammonium formate, $90 \%$ aqueous acetonitrile, $\mathrm{pH}, 10.0$ ) for 40 minutes and increased from $5 \%$ to $30 \%$ linearly at a flow rate of $0.3 \mathrm{~mL} / \mathrm{min}$. Each tube was collected every 1 minute for a total of 40 tubes. Four tubes were combined into one component from the first tube, and 10 in total were obtained. The components were subjected to centrifugal drying for LC-MS analysis.

\section{Reverse phase nanoflow HPLC and tandem mass spectrometry}

Reversed-phase nano-LCMS/MS analysis was performed on an Eksigent NanoLC-UltraTM 2D system (AB SCIEX, Concord, ON, USA). The isolated polypeptide samples were redissolved in $30 \mu \mathrm{L}$ of $2 \%$ acetonitrile, and $0.1 \%$ formic acid, with $10 \mu \mathrm{L}$ of the sample taken and loaded into a ChromXP C18 (3 $\mu \mathrm{m}, 120 \AA$ ) NanoLC trap. Desalting was carried out for 10 minutes at a rate of $2 \mu \mathrm{L} / \mathrm{min}$ using solution A $(0.1 \%$ formic acid, $2 \%$ acetonitrile, $98 \%$ aqueous solution). Gradient elution with solution B (0.1\% formic acid, $2 \%$ water, $98 \%$ acetonitrile) was performed using a $5-40 \%$ linear gradient of solution $\mathrm{B}$ on the analytical column $\left(75 \mu \mathrm{m}^{*} 15 \mathrm{~cm}, \mathrm{C} 18,3 \mu \mathrm{m}\right.$, $120 \AA$ A, ChromXP Eksigent). Elution was undertaken, and the time was controlled to within 70 minutes. LC-MS/MS analysis was performed on a TripleTOF 5600+ System (AB SCIEX, Concord, ON, USA) equipped with a Nanospray III ion source (AB SCIEX, Concord, ON, USA). Data were obtained using a $2.4 \mathrm{kV}$ ion spray voltage, 30 PSI curtain gas, 5 PSI atomizing gas, and a $150{ }^{\circ} \mathrm{C}$ interface heater temperature. For the IDA scan mode, the mass spectrum scan range was $400-1,250 \mathrm{~m} / \mathrm{z}$, and the primary scan was accumulated for $250 \mathrm{~ms}$ if the parent ion signal strength was greater than 200 counts per second (counts/s) and the charge was +2 to +5 . The secondary mass spectrometry collision energy was automatically adjusted, and the dynamic exclusion was set for $1 / 2$ of the peak width $(\sim 16 \mathrm{~s})$.

\section{Protein identification and quantification}

Raw data from the mass spectra were processed using ProteinPilot Software v. 4.5 (Sciex Inc., USA), and protein identification and relative quantitative analysis were performed with a false positive rate set to $0.1 \%$. The database search parameters were set using iTRAQ 8-plex peptide labeling quantification. The database of the assembled Arachis hypogaea genome contains 38,967 sequences, trypsin digestion, and depth analysis mode. The mass error of primary and secondary mass spectra was $20 \mathrm{ppm}$ and $0.1 \mathrm{Da}$, respectively. There was a $95 \%$ confidence threshold at the protein level (unused protein score $>1.3$ ). For proteins to be determined to be differentially expressed, the fold change had to be greater than 1.2 or less than 0.83 , and the $\mathrm{P}$ value should be less than 0.05 .

\section{RT-PCR}

The mRNA was extracted via SteadyPure Universal RNA Extraction Kit (Accurate biology, AG21017, China), and cDNA was synthesized using PrimeScript RT Reagent Kit with gDNA Eraser (Takara, RR047A, Japan). RTPCR was performed using TB Green Premix Ex Taq II (Takara, RR820B, Japan) for quantitative assessment of the expression of the target genes. We selected 5 proteins in each of the top 20 increased, and the top 20 decreased DEPs and found the following mRNA. The primer sequence is as follows: Dnase1 (forward, 5'-ATTGCTGTGGTCCAAGAGGTC-3' and reverse, 5'-AGCGATAGTTGTCAGGGATGTC-3'), LBHD2 (forward, 5'-GGAAGGAAGCAGATGATGATGTC-3' and reverse, 5'-TGCCACGCCCTTATCTGTTAC-3'), Loxhd1, (forward, 5' - AGAAGAAGAAGCGGCGGA AG-3' and reverse, 5'-TTCGTGCTCCAACTCAT CCTC-3'), FCGBP, (forward, 5'-CAAAGTCTTCCC TAATGGCACAG-3' and reverse, 5'-ATACTCC 
TCCACTCGGTCCTC-3'), EML5, (forward, 5'-TA TCTGCCCATCTGTGCTACTG-3' and reverse, 5 '-CGGACTGCCAACATACAATGAC-3'), DMN, (forward, 5'-ACCTCCATCCAGCACATTGAC-3' and reverse, 5'-TGACCTCTCCACCAGGACTAC-3'), GIPC3, (forward, 5'-AGGACGCTCTAGGACTGACC-3' and reverse, 5'-CCTGTTGATAATGCTGCCTTCC-3'), PHACTR3, (forward, 5'-CCTCATCTCACCACT GTCCAC-3' and reverse, 5'-GCACATCCA TCCGCTTCTTTG-3'), Hba-a3, (forward, 5'-CTGCTGGTGACCTTGGCTAG-3' and reverse, 5'-CTTGGAAGTCAACACAGTGCTC-3'), and Mcpt2, (forward, 5'-CATCTGTGGTGGGTTTCTCATAAG-3' and reverse, 5'-GCTGTGTGGATTCTCGCTTTC-3').

\section{Immunoblotting}

After cutting the bladder tissue into pieces with scissors, it was placed in a homogenizer. A mixture of NP40 lysate was added (Beyotime, P0012F, China), then 1\% protease inhibitor (Roche, 4693159001, Switzerland), and was homogenized repeatedly until there was no obvious precipitation. The tissue was then centrifuged at $12,000 \mathrm{rpm}$ at $4{ }^{\circ} \mathrm{C}$ for $5 \mathrm{~min}$, with the supernatant being extracted. The protein concentration was determined by a BCA reagent test kit (Beyotime, P0010, China). The protein sample $(30 \mu \mathrm{g})$ was separated by SDS-PAGE gel and transferred to a PVDF membrane and blocked for 1 hour, incubated with collagen I (Abcam, ab254113, America, rabbit, 1:1,000), mast cell protease 1 (Affinity, DF12290, China, rabbit, 1:1,000), AOAH (Affinity, DF9158, China, rabbit:cat 1:1,000), GAPDH (Abcam, ab8245, America, mouse, 1:5,000), and $\beta$ - actine (Abcam, ab6276. America, mouse, 1:5,000) overnight at $4{ }^{\circ} \mathrm{C}$. Membranes were labeled with the appropriate secondary antibody and visualized by enhanced chemiluminescence. Quantitative analysis of blots was performed by densitometry using Image $\mathrm{J}$ software.

\section{Statistical analysis}

The measurement data were expressed as mean \pm standard deviation $(\bar{x} \pm s)$. Data were analyzed by GraphPad 8 statistical software using the Student's $t$-test or one-way ANOVA. iTRAQ labeling was performed for 3 biological replicates in each group, and the statistical significance was defined as $\mathrm{P}<0.05$.

\section{Results}

\section{Pathological changes of the bladder in ketamine-induced rat models}

One rat in the experimental group died after being injected with ketamine. The rat's cause of death was thought to be due to the inhibitory effect of ketamine on breathing. After ketamine treatment for 8 weeks, the rat bladders were evaluated through HE staining (Figure 1A,B). Bladder epithelia from the experimental group was significantly thinner (blue arrow; Figure 1B). Arterial dilatation accompanied by congestion (black arrow; Figure $1 B$ ), and infiltration of more inflammatory cells (yellow arrow; Figure 1B) were also observed in the ketamine group. Masson's trichome staining showed that the deposition of collagen fibers in the bladder submucosal and intermuscular bundles were increased in the experimental group (Figure 1C,D). Histology results indicated that ketamine-induced urothelial thinning, blood congestion, inflammatory infiltration, and fibrosis were present in the bladders.

\section{Elevated collagen I expression after ketamine treatment}

As an important indicator to evaluate the occurrence of fibrosis $(11,12)$, the expression of the collagen I protein was significantly increased in the experimental group $(\mathrm{P}=0.0181)$ (Figure 2). The results show that after 8 weeks of tail vein injection, obvious fibrosis had occurred in the rat bladders.

\section{Differences of protein expression in the bladder after ketamine treatment}

A total of 3,690 proteins were identified by protein analysis using iTRAQ. The protein data were quality-controlled in four aspects: protein extraction, proteolysis, mass spectrometry, and proteomic identification depth assessment (Appendix 1: Figure S1). A protein expression difference greater than 1.2-fold, and a statistical $\mathrm{P}$ value of $\mathrm{T}$ test less than 0.05 was considered to be indicative of differentially expressed proteins (DEPs), resulting in a total of 727 DEPs, including 423 differentially upregulated proteins (DUPs) and 304 differentially down-regulated proteins (DDPs). A hierarchical cluster analysis of DEPs was then performed. The heat map visually showed changes in protein expression levels in both the experimental and control groups (Figure $3 A$ ), and principal component analysis (PCA) was 

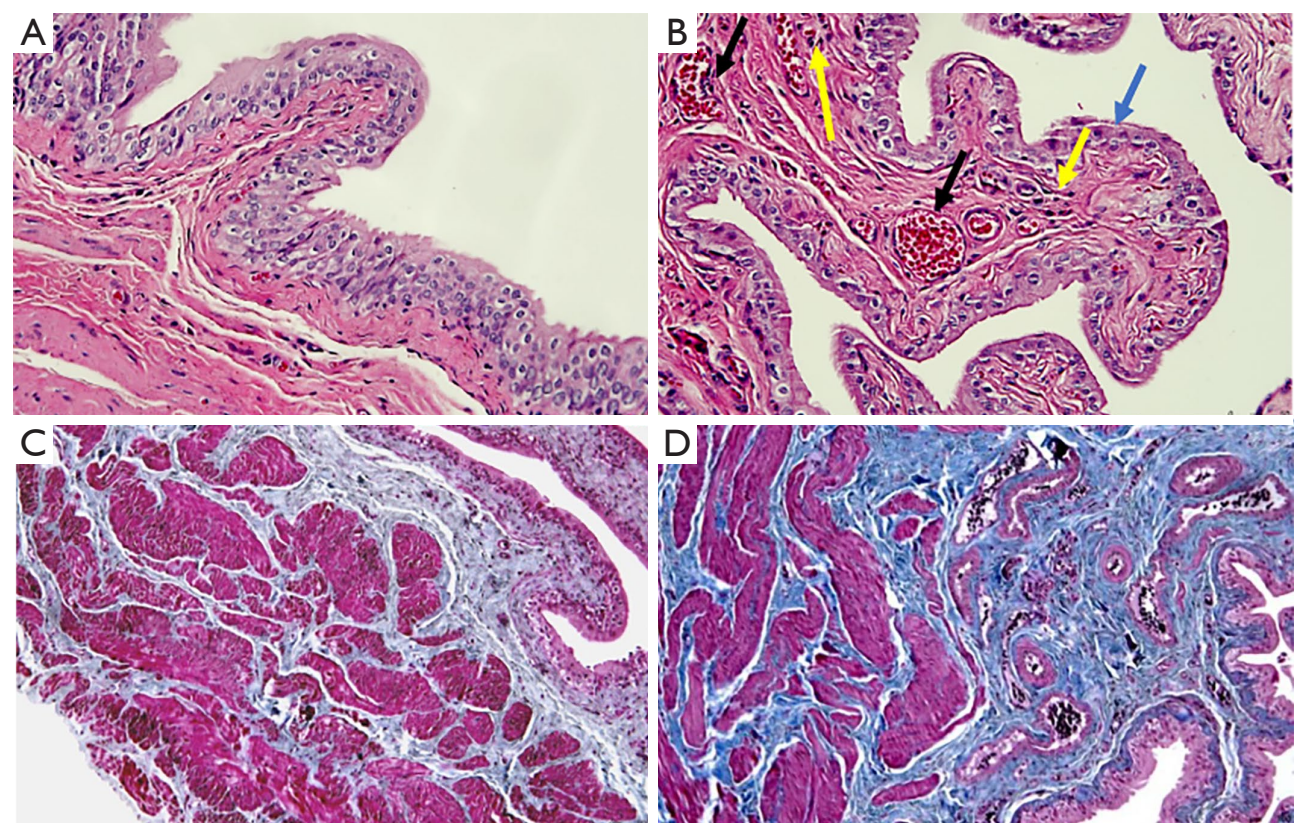

Figure $1 \mathrm{HE}$ staining and Masson's trichrome staining of rat bladder tissue. (A) HE staining of rat bladder tissue in the control group (magnification, $\times 200$ ), (B) HE staining of rat bladder in the ketamine-treated group following 8 weeks of treatment (magnification, $\times 200$ ). Bladder epithelia from the experimental group was significantly thinner (blue arrow). Arterial dilatation accompanied with congestion (black arrow) and infiltration of more inflammatory cells (yellow arrow), (C) the control group (magnification, $\times 100$ ), (D) Ketamine-treated rat following 8 weeks of treatment (magnification, $\times 100$ ). Shows a large collagen deposit.

A

Control Ketamine

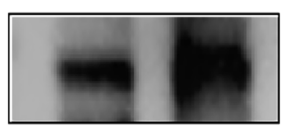

anti-collagen I

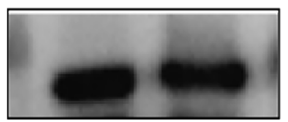

anti-gapdh
B

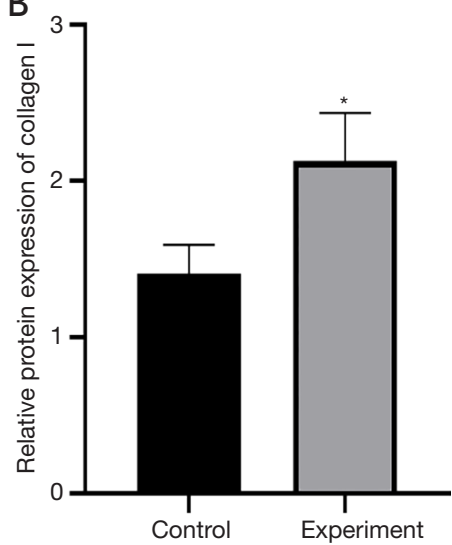

Figure 2 The expression of collagen I in rat bladders increased after ketamine treatment. (A) Representative images of western blot analysis examining the collagen I expression in rat bladders. (B) Quantitative densitometric analysis of the ratio of collagen I versus GAPDH control. The expression of collagen $\mathrm{I}$ in the experimental group was significantly higher than in the control group, $\mathrm{P}=0.0181$. Results are expressed as the mean \pm s.e.m. obtained from seven mice. *, $\mathrm{P}<0.05$. 

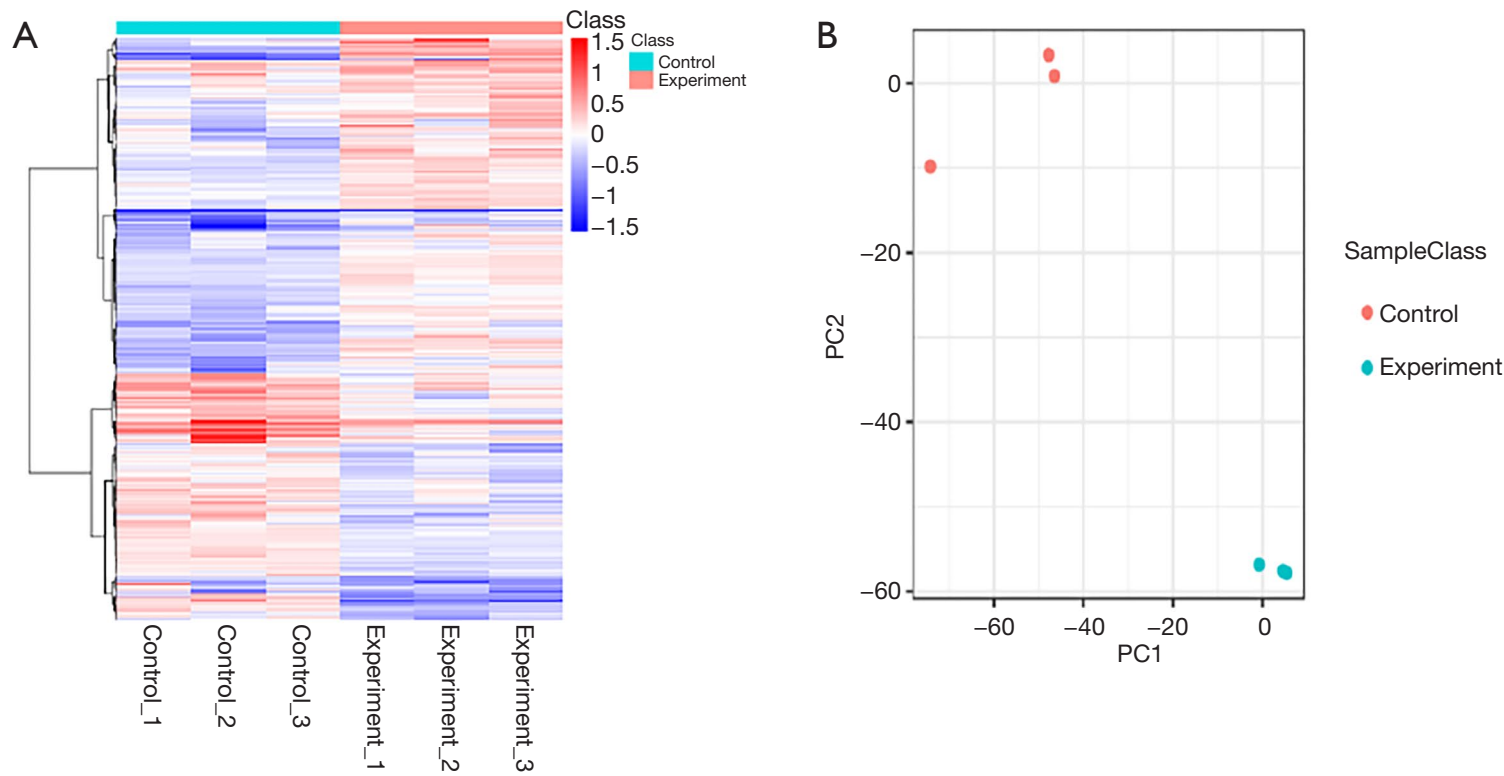

Figure 3 Overall situation of DEPs. (A) Heat map of DEPs ratio, each row represents a protein, each column represents the ratio of a sample to a reference sample, and the ratio takes the value of $\log 2$. (B) Principal component analysis of DEPs. Experimental group and control group have a good differentiation. DEP, differential expression protein.

further performed to identified DEPs (Figure 3B).

\section{Verification of the proteomics results by RT-PCR and WB}

Among the top 20 increased and the top 20 decreased DEPs (Appendix 2: Table S1, Table S2), the 10 selected mRNA, and the expression of DNase I, LBDH2, LOXHD1, FCGBP, DMN, GIPC3, PHACTR3, Hba-a3, and Mcpt2 were consistent with the proteomics results (Figure 4A). The expression of AOAH and mast cell (MC) protease 1 protein in the experimental group decreased (Figure $4 B$ ). These results are generally consistent with the results of proteomics, which proves the credibility of the data.

\section{Functional enrichment analysis of DEPs}

We made use of volcano maps to show univariate statistical analysis results. The abscissa is the ratio of the mean value of the two phenotypes, and the ordinate was the $\mathrm{P}$ value (represented by $-\log 10)$ corrected by the statistical test (Figure 5A). The intra-group difference analysis of the biological replicate samples by the variation $(\mathrm{CV})$ coefficient showed that the protein $\mathrm{CV}$ of more than $75 \%$ of the samples was less than $20 \%$ (Figure $5 A$ ). We also took advantage of GO, KEGG, COG, and EggNOG databases to analyze the function of the DEPs. The comparison results of DEPs using COG and EggNOG databases and statistics of entries subcellular localization and transcription factors are found in Appendix 3 (Figure S2).

The GO function classification of DEPs mainly includes biological progress, cellular components, and molecular function. In biological progress, proteins mainly involve biological regulation (436 proteins), regulation of cellular processes (377 proteins), response to stimuli (321 proteins), cellular components mainly involving cytoskeletons (120 proteins), plasma membrane segments (109 proteins), and cytoskeletal segments (94 proteins). The molecular function was mainly related to transition metal ion binding (64 proteins) and structural molecule activity (64 proteins) (Figure 5B). Enrichment factor analysis was carried out to reflect the degree and significance of enrichment, and hypergeometric tests on the GO of the differential protein were performed to obtain enriched GO entries, and it showed the top 20 functional entries (Figure 5B).

In the KEGG analysis of DEPs, a total of 287 major KEGG pathways were detected, with 15 significantly changed KEGG pathways ( $\mathrm{P}$ values $<0.05$ ). All pathways belonged to six major categories, cellular processes, environmental information processing, genetic information processing, human diseases, metabolism, and organismal 

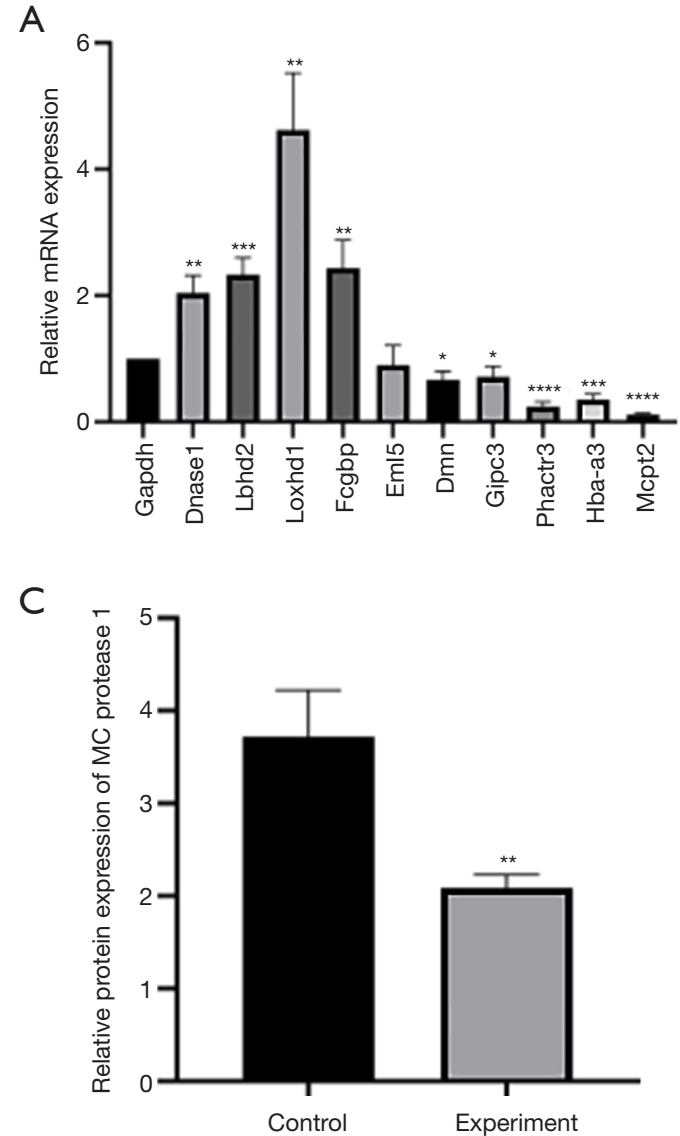

B
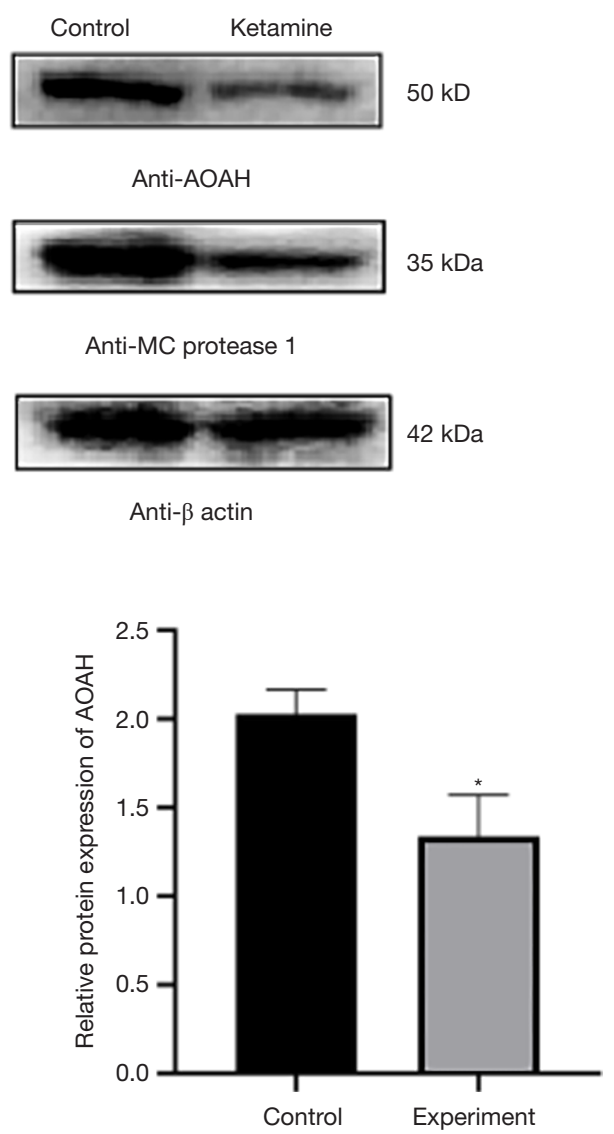

Figure 4 Proteomics results verification by RT-PCR and WB. (A) Verification results of the 10 selected mRNA. The expression of DNase I, LBDH2, LOXHD1, FCGBP, DMN, GIPC3, PHACTR3, Hba-a3, and Mcpt2 were consistent with the proteomics results. (B) Representative images of western blot analysis examining $\mathrm{AOAH}$ and $\mathrm{MC}$ protease 1 expression in rat bladders. The expression of AOAH and $\mathrm{MC}$ protease 1 in the experimental group decreased compared to the control group. (C) Quantitative densitometric analysis of the ratio of AOAH versus GAPDH loading control. The experimental group was significantly reduced, $\mathrm{P}=0.0117$. (D) Quantitative densitometric analysis of the ratio of $\mathrm{MC}$ protease 1 versus $\mathrm{GAPDH}$ loading control. The experimental group was significantly reduced, at $\mathrm{P}=0.0056$. Results are expressed as the mean \pm SEM. * $, \mathrm{P}<0.05,{ }^{* *}, \mathrm{P}<0.01$.

systems. In contrast, the top three pathways for protein participation are signal transduction (100 proteins), cancers: overview (83 proteins), and global and overview maps (82 proteins) (Figure 5C). We performed a hypergeometric test on the KEGG of DEPs. The top three KEGGs had significant changes to the metabolism of xenobiotics by cytochrome $\mathrm{P} 450$, chemical carcinogenesis, and drug metabolism - cytochrome P450 (Figure 5C).

\section{Discussion}

Bladder fibrosis induced by ketamine is irreversible, causing great distress to patients and doctors. The purpose of this research was to explore the mechanism of bladder fibrosis. In the present study, a model of ketamine-induced bladder fibrosis through tail vein injection was established. Protein expression changes were then quantitatively detected in rat bladders using proteomic technology. The expression of MC protease 1 and $\mathrm{AOAH}$ was significantly reduced, and the triggering of the platelet activation pathway was found through the use of bioinformatics technologies, suggesting that they may play different regulatory roles in ketamineinduced bladder fibrosis.

Clinically, patients with long-term abuse of ketamine will have a contracted bladder, but there is no relevant literature available that successfully establishes a contracted bladder 

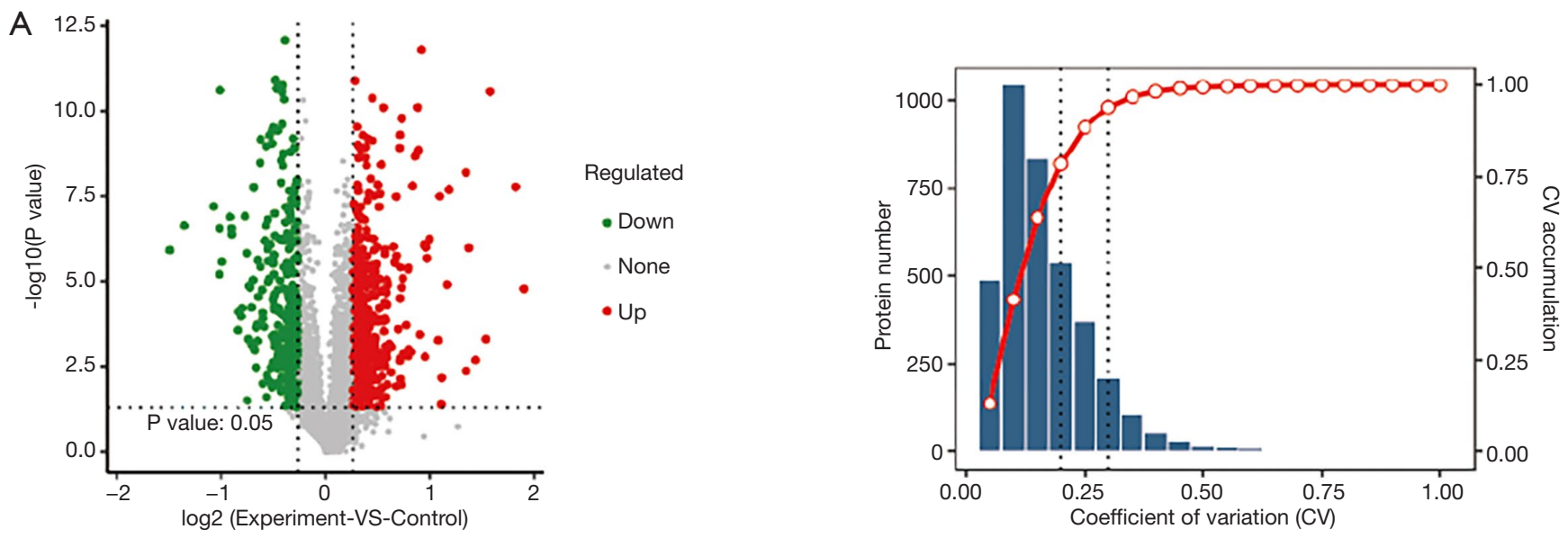

B
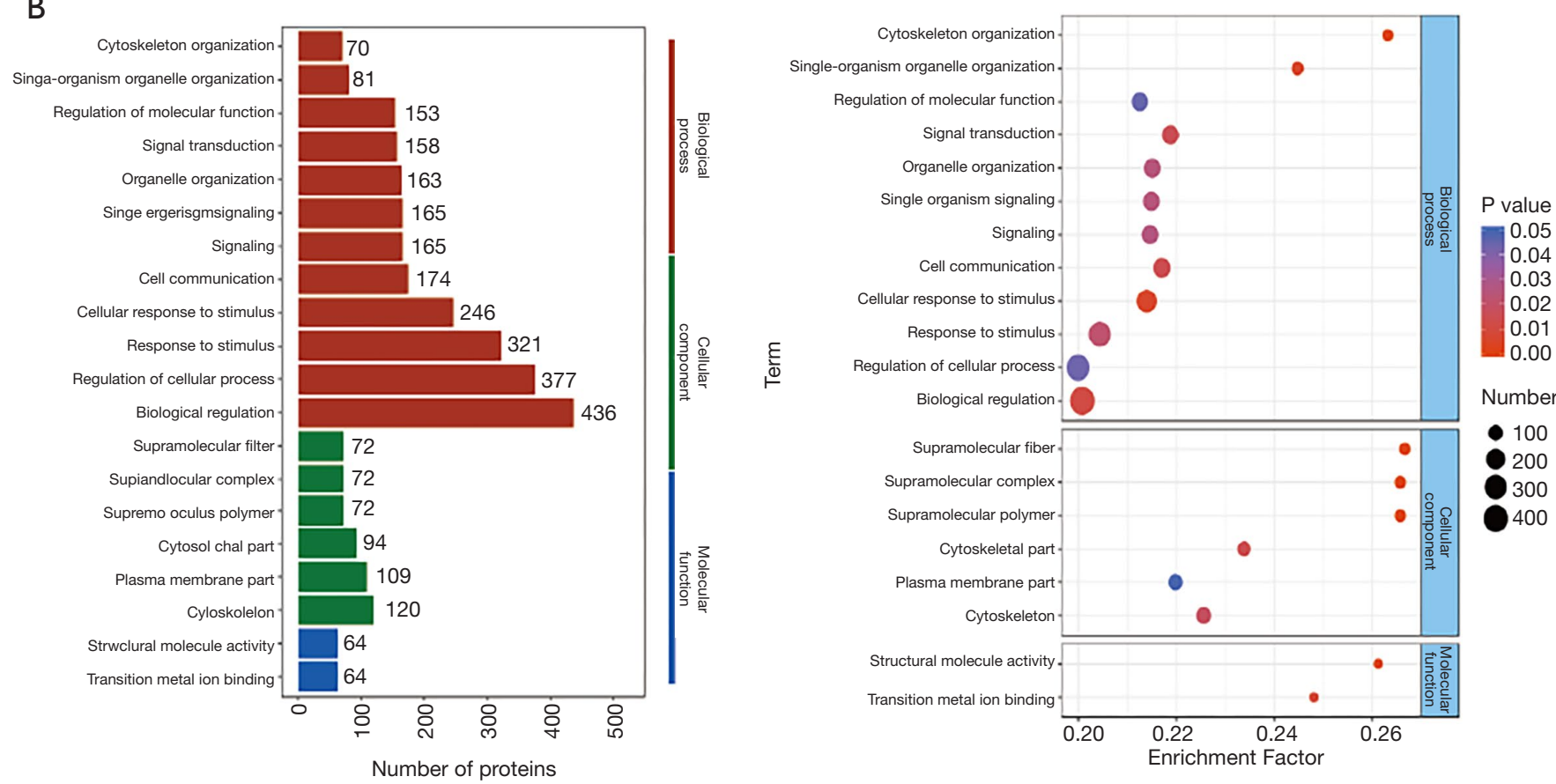

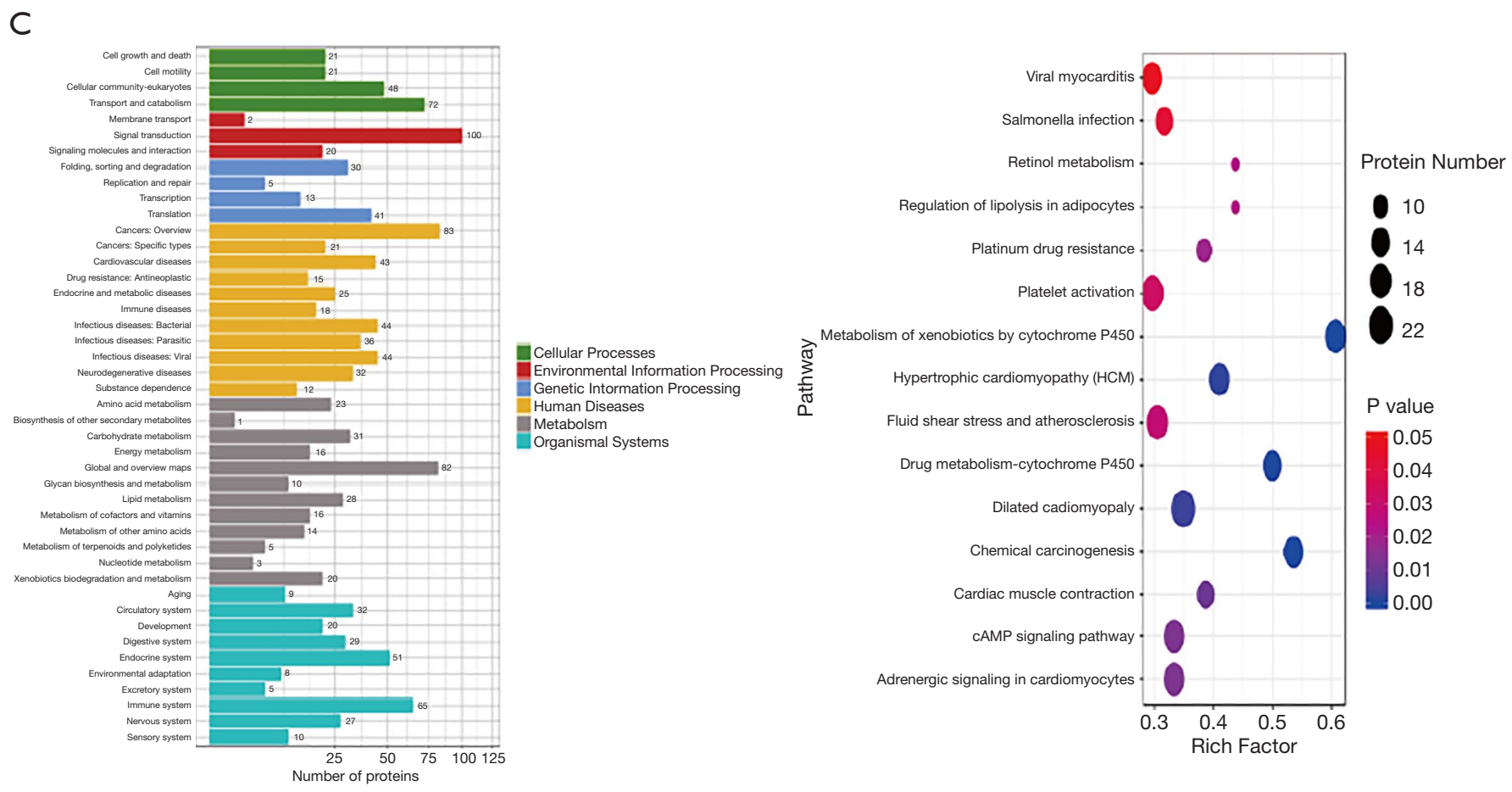

Figure 5 Quantitative quality control and functional annotation of DEPs. (A) Volcano map and CV distribution map. In the volcano map, red dots represent up-regulation, blue dots represent down-regulation, including 423 differentially upregulated proteins and 304 differentially down-regulated proteins. In the $\mathrm{CV}$ distribution map, the protein $\mathrm{CV}$ of more than $75 \%$ of the samples was less than $20 \%$, which indicates that the difference of biological repetition was small. (B) GO entry and GO enrichment factor of DEPs. The GO classification chart shows the distribution of the items involved in cellular components, biological processes, and molecular function. Enrichment factor refers to the ratio of the number of proteins located in this functional entry among DEPs to the total number of proteins located in this entry in all annotated proteins. The larger the enrichment factor, the greater the degree of enrichment. $\mathrm{P}$ value ranges from 0 to 1 . The closer to zero, the more significant the enrichment. (C) DEPs pathway entry and KEGG enrichment factors of DEPs. The annotation results of the differentially expressed gene from KEGG are classified according to the type of pathway in KEGG. DEP, differential expression protein.

model in animal experiments. In the study by Song et al., they injected different concentrations of ketamine through the tail vein for two weeks. They found that the amount of bladder collagen deposition increased with greater concentrations of ketamine, and their team continued to use a $25 \mathrm{mg} / \mathrm{kg}$ ketamine tail vein injection for two weeks to study ketamine-induced bladder fibrosis $(5,13)$. However, this team injected ketamine for a relatively short time, which cannot present the same clinical situation as longterm ketamine use. Therefore, we extended the ketamine injection time to 8 weeks to better meet the clinical situation.

In HE staining, inflammatory cell infiltration under the bladder mucosa and thinning of the bladder epithelial layer could be seen, which was different from previous research that indicated the bladder urothelium had not changed or even thickened $(5,13)$. These differences are thought to be due to the treatment of ketamine by tail vein injection rather than an intraperitoneal injection and the extended duration of treatment. Therefore, the effect of ketamine on the bladder urothelium of rats was stronger in this study compared to the Song study. Most scholars believe that bladder epithelial barrier dysfunction is an important pathogenic mechanism of KC (14). The results of this paper's study suggest that ketamine causing damage to the bladder urothelium is consistent with the above view. Previous studies indicated that bladder fibrosis without urothelium developed in rats treated with ketamine for 2 weeks $(5,13)$. However, further investigations of interstitial bladder components are required to clarify their 
effects in the pathogenesis of urothelial damages.

In rats treated with ketamine, bladder fibrosis was confirmed through Masson's trichrome staining and collagen I protein expression level detection. In this study, after confirming the occurrence of ketamineinduced bladder fibrosis, proteomics-based on iTRAQ technology was used to identify DEPs. A total of 3,690 proteins were identified, and 727 different protein expressions were greater than 1.2 times above normal levels. Previously, Gu et al. used two-dimensional gel electrophoresis to study the effects of long-term ketamine injections on rat bladder protein expression, and a total of about 1,200 proteins were identified (15). The proteins identified by iTRAQ technology were of greater quantity and more accurate (8).

To investigate the possible mechanism of bladder fibrosis, GO, KEGG databases were used to predict the function of DEPs. In the KEGG map, 65 proteins with differential expressions belonged to the immune system, and 18 of them were involved in immune diseases, suggesting that immunomodulation may play an important role in ketamine-induced bladder disease. Tissueinfiltrating immune cells can inhibit fibrosis by releasing various inflammatory inhibitors (16). As granulated cells, MC can release a variety of inflammatory mediators, including proteases (17). At present, most research focuses on mast cell protease 4 , and indicates that it shows both anti-inflammatory and pro-inflammatory effects (17). In the renal tubulointerstitial fibrosis mouse model, MC can release $\mathrm{MC}$ proteases 4 to limit fibrosis development (18). As for other isoforms, little is known about them relative to the process of fibrosis. This study's results show the significantly decreased expression of MC protease 1 . It has been proved that MC proteases can cause degradation of type VI collagen and procollagen $(19,20)$. Although the specific functions of MC protease 1 are not clear, it is the protein with the most significant reduction after rat bladder fibrosis, and its isomer has the capability to inhibit the process of fibrosis. Therefore, this study suggests that MC protease 1 may play an important regulatory role by acting as factor of inhibiting fibrosis in the process of ketamineinduced bladder fibrosis.

As a neutrophil lipase, AOAH has the ability to attenuate inflammation by selectively releasing secondary fatty acyl chains $(21,22)$. Altered AOAH expression is associated with chronic inflammatory diseases, such as ulcerative colitis (23). Compared with wild-type mice, AOAH knockout mice have higher rates of the neuronal firing of bladder afferents, increased bladder permeability, more mast cell infiltration, and vascular endothelial growth factor expression in bladder tissue $(24,25)$. These changes are similar to those of interstitial cystitis (IC). KC has many similarities in clinical symptoms and bladder pathology with IC (26). In this study, the expression of AOAH decreased significantly after being treated with ketamine in the rat bladder tissue. It is suggested that the decrease of $\mathrm{AOAH}$ may be related to $\mathrm{KC}$ symptoms such as bladder inflammation, overactive bladder, and pelvic pain. However, the study also found that enhanced bladder capacity and larger void volumes are seen in $\mathrm{AOAH}$ deficient mice $(24,25)$. The function of $\mathrm{AOAH}$ in $\mathrm{KC}$ is not clear and needs to be confirmed by further investigation.

A total of 64 proteins in the platelet activation pathway were detected, and 19 proteins were expressed differently (Appendix 4: Table S3, Table S4, Figure S3). The collagen expression was elevated in rat bladders treated with ketamine, indicating that the platelet activation pathway was triggered and prompted aggregation of fibrinogen in ketamine-induced bladder dysfunction. It has been thought that platelets mainly participate in hemostasis and thrombosis. It was now known that platelets and their activation products also play an important role in immune and inflammatory processes (27). Miao found that platelets are activated in patients with ANCA-associated vasculitis, and the activated platelet can trigger an alternative complement pathway (28). Platelets directly activate the complement system and interact with white blood cells and secrete various substances after activation to promote the progression of inflammation (29). Platelet-activating factor (PAF) is a biologically active lipid transmitter originally found in the activated platelet aggregation. In subsequent studies, PAF was found to play an important role in various inflammatory disorders such as asthma, peritonitis, and systemic allergic reactions $(27,30)$. Multiple PAF isoforms and PAF-like lipids can cause IL-1 $\beta$ and IL-18 to mature by activating inflammatory bodies and promoting inflammation $(27,30)$. In the results of this study's HE staining, a large amount of inflammatory cell infiltration was observed. Triggering of the activated platelet pathway causes platelet activation and the secretion of various active substances, acting on the effector cells of inflammation, causing an imbalance of the extracellular matrix (increased dynamic deposition or insufficient resorption), thereby promoting the occurrence of bladder fibrosis. 


\section{Conclusions}

After a rat model of the ketamine-induced bladder fibrosis was successfully assembled, iTRAQ protein mass spectrometry was used to study the mechanism of ketamineinduced bladder fibrosis. Through the study of DEPs, it was found that the expression of MC protease 1 decreased, and the activation of the platelet activation pathway suggests that they may play an important role in the mechanism of ketamine-induced bladder fibrosis.

\section{Acknowledgments}

The authors wish to acknowledge Teacher Zhou from the Experimental Animal Center of Central South University for animal experiment operation guidance. Acknowledgments to Shenzhen Wininnovate Bio-Tech for technological support and J. Collie and J. Chapnick for English modification.

Funding: This work was supported by the National Natural Science Foundation of China (Grant No. 81700667).

\section{Footnote}

Reporting Checklist: The authors have completed the ARRIVE reporting checklist. Available at https://dx.doi. org/10.21037/tau-21-351

Data Sharing Statement: Available at https://dx.doi. org/10.21037/tau-21-351

Conflicts of Interest: All authors have completed the ICMJE uniform disclosure form (available at https://dx.doi. org/10.21037/tau-21-351). The authors have no conflicts of interest to declare.

Ethical Statement: The authors are accountable for all aspects of the work in ensuring that questions related to the accuracy or integrity of any part of the work are appropriately investigated and resolved. Experiments were performed under a project license (No. 2018sydw0225) granted by the Experimental Animal Welfare Center of Central South University, Hunan, China, in compliance with China's "Animal management regulations" for the care and use of animals.

Open Access Statement: This is an Open Access article distributed in accordance with the Creative Commons
Attribution-NonCommercial-NoDerivs 4.0 International License (CC BY-NC-ND 4.0), which permits the noncommercial replication and distribution of the article with the strict proviso that no changes or edits are made and the original work is properly cited (including links to both the formal publication through the relevant DOI and the license). See: https://creativecommons.org/licenses/by-nc-nd/4.0/.

\section{References}

1. Sihra N, Ockrim J, Wood D. The effects of recreational ketamine cystitis on urinary tract reconstruction - a surgical challenge. BJU Int 2018;121:458-65.

2. Jhang JF, Hsu YH, Jiang YH, et al. Histopathological characteristics of ketamine-associated uropathy and their clinical association. Neurourol Urodyn 2018;37:1764-72.

3. Shahani R, Streutker C, Dickson B, et al. Ketamineassociated ulcerative cystitis: a new clinical entity. Urology 2007;69:810-2.

4. Huang LK, Wang JH, Shen SH, et al. Evaluation of the extent of ketamine-induced uropathy: the role of CT urography. Postgrad Med J 2014;90:185-90.

5. Song M, Yu HY, Chun JY, et al. The fibrosis of ketamine, a noncompetitive $\mathrm{N}$-methyl-d-aspartic acid receptor antagonist dose-dependent change in a ketamine-induced cystitis rat model. Drug Chem Toxicol 2016;39:206-12.

6. Wang J, Chen Y, Gu D, et al. Ketamine-induced bladder fibrosis involves epithelial-to-mesenchymal transition mediated by transforming growth factor- $\beta 1$. Am J Physiol Renal Physiol 2017;313:F961-72.

7. Cui YH, Liu Q, Xu ZY, et al. Quantitative proteomic analysis of human corneal epithelial cells infected with HSV-1. Exp Eye Res 2019;185:107664.

8. Casado-Vela J, Martínez-Esteso MJ, Rodriguez E, et al. iTRAQ-based quantitative analysis of protein mixtures with large fold change and dynamic range. Proteomics 2010;10:343-7.

9. Moodley YP, Corte TJ, Oliver BG, et al. Analysis by proteomics reveals unique circulatory proteins in idiopathic pulmonary fibrosis. Respirology 2019;24:1111-4.

10. Cao W, Li Y, Li M, et al. Txn1, Ctsd and Cdk4 are key proteins of combination therapy with taurine, epigallocatechin gallate and genistein against liver fibrosis in rats. Biomed Pharmacother 2017;85:611-9.

11. Zhang Y, Zhang R, Han X. Disulfiram inhibits inflammation and fibrosis in a rat unilateral ureteral obstruction model by inhibiting gasdermin D cleavage and 
pyroptosis. Inflamm Res 2021;70:543-52.

12. Sari DCR, Budiharjo S, Afifah H, et al. Centella asiatica Extract Attenuates Kidney Fibrosis Through Reducing Mesenchymal Transition and Inflammation in Ureteral Ligation Model in Mice. Front Pharmacol 2021;12:621894.

13. Kim A, Yu HY, Heo J, et al. Mesenchymal stem cells protect against the tissue fibrosis of ketamine-induced cystitis in rat bladder. Sci Rep 2016;6:30881.

14. Shen CH, Wang ST, Lee YR, et al. Biological effect of ketamine in urothelial cell lines and global gene expression analysis in the bladders of ketamine-injected mice. Mol Med Rep 2015;11:887-95.

15. Gu D, Huang J, Shan Z, et al. Effects of long-term ketamine administration on rat bladder protein levels: a proteomic investigation using two-dimensional difference gel electrophoresis system. Int J Urol 2013;20:1024-31.

16. Pejler G, Rönnberg E, Waern I, et al. Mast cell proteases: multifaceted regulators of inflammatory disease. Blood 2010;115:4981-90.

17. Caughey GH. Mast cell tryptases and chymases in inflammation and host defense. Immunol Rev 2007;217:141-54.

18. Beghdadi W, Madjene LC, Claver J, et al. Mast cell chymase protects against renal fibrosis in murine unilateral ureteral obstruction. Kidney Int 2013;84:317-26.

19. Kielty CM, Lees M, Shuttleworth CA, et al. Catabolism of intact type VI collagen microfibrils: susceptibility to degradation by serine proteinases. Biochem Biophys Res Commun 1993;191:1230-6.

20. Kofford MW, Schwartz LB, Schechter NM, et al. Cleavage of type I procollagen by human mast cell chymase initiates collagen fibril formation and generates a unique carboxylterminal propeptide. J Biol Chem 1997;272:7127-31.

21. Lu M, Varley AW, Ohta S, et al. Host inactivation of bacterial lipopolysaccharide prevents prolonged tolerance following gram-negative bacterial infection. Cell Host Microbe 2008;4:293-302.

22. Staab JF, Ginkel DL, Rosenberg GB, et al. A saposin-like domain influences the intracellular localization, stability, and catalytic activity of human acyloxyacyl hydrolase. J Biol Chem 1994;269:23736-42.

23. Fehrmann RS, Jansen RC, Veldink JH, et al. Trans-eQTLs reveal that independent genetic variants associated with a complex phenotype converge on intermediate genes, with a major role for the HLA. PLoS Genet 2011;7:e1002197.

24. Yang W, Yaggie RE, Jiang MC, et al. Acyloxyacyl hydrolase modulates pelvic pain severity. Am J Physiol Regul Integr Comp Physiol 2018;314:R353-65.

25. Aguiniga LM, Searl TJ, Rahman-Enyart A, et al. Acyloxyacyl hydrolase regulates voiding activity. Am J Physiol Renal Physiol 2020;318:F1006-16.

26. Lee CL, Jiang YH, Kuo HC. Increased apoptosis and suburothelial inflammation in patients with ketaminerelated cystitis: a comparison with non-ulcerative interstitial cystitis and controls. BJU Int 2013;112:1156-62.

27. Chen X. Rac1 regulates platelet microparticles formation and rheumatoid arthritis deterioration. Platelets 2020;31:112-9.

28. Miao D, Li DY, Chen M, et al. Platelets are activated in ANCA-associated vasculitis via thrombin-PARs pathway and can activate the alternative complement pathway. Arthritis Res Ther 2017;19:252.

29. Miao D, Ma TT, Chen M, et al. Platelets release proinflammatory microparticles in anti-neutrophil cytoplasmic antibody-associated vasculitis. Rheumatology (Oxford) 2019. [Epub ahead of print]. doi: 10.1093/ rheumatology/kez044.

30. Ishii S, Shimizu T. Platelet-activating factor (PAF) receptor and genetically engineered PAF receptor mutant mice. Prog Lipid Res 2000;39:41-82.
Cite this article as: Zhu Q, Li H, Li K, Wang Z, Tang Z. Proteomic analysis exploring the mechanism of bladder fibrosis induced by ketamine using a rat model. Transl Androl Urol 2021;10(8):3300-3311. doi: 10.21037/tau-21-351 


\section{Supplementary}

\section{Appendix 1}

$84.12 \%$ of the proteins were between $0-100 \mathrm{kDa}$ (Figure S1A). After proteolysis by trypsin, $95.1 \%$ of the protein could be detected by mass spectrometry, indicating that the enzymatic efficiency was extraordinarily high (Figure S1A). In general, the peptides of the protein profile are dominated by $2+$ and $3+$, and in our study the peptides of $2+$ and $3+$ accounted for $81.2 \%$ (Figure $S 1 A$ ). For the spectra and peptides identified to the protein, it is generally believed that the greater the number, the higher the confidence of the protein. In this result, $68.83 \%$ of the proteins had more than 2 spectra to support the identification, and $60.68 \%$ of the proteins had 2 unique peptides to support the identification (Figure S1B). We used the target-decoy method for false positive evaluation. The protein identification threshold was false discovery rate $(\mathrm{FDR})<1 \%$, and the FDR of the peptide-spectrum match (PSM) and protein were evaluated separately (Figure S1B).

A
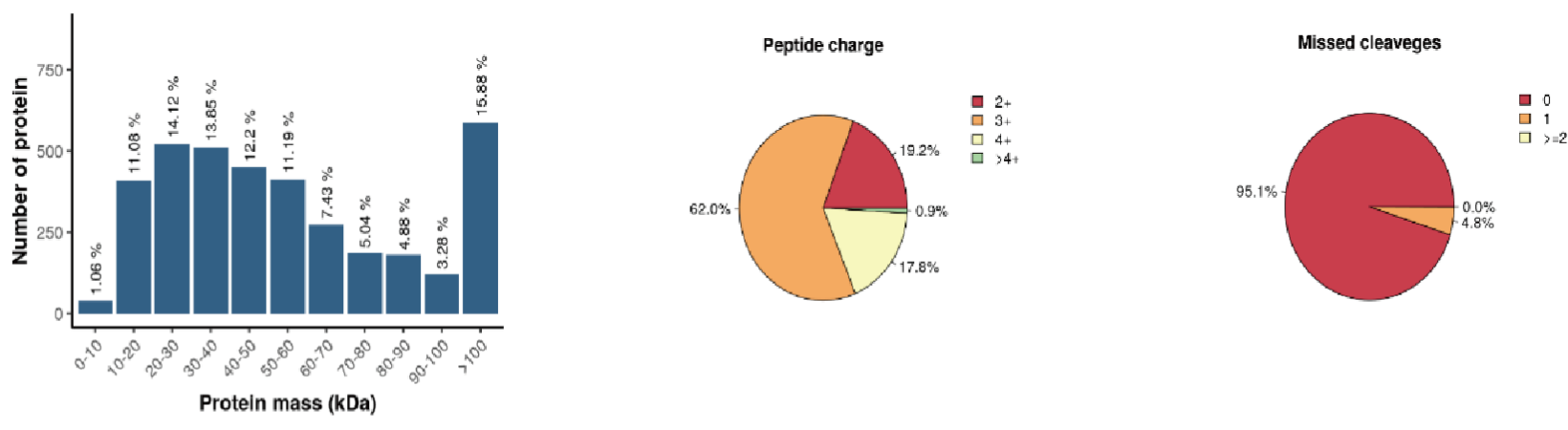

B
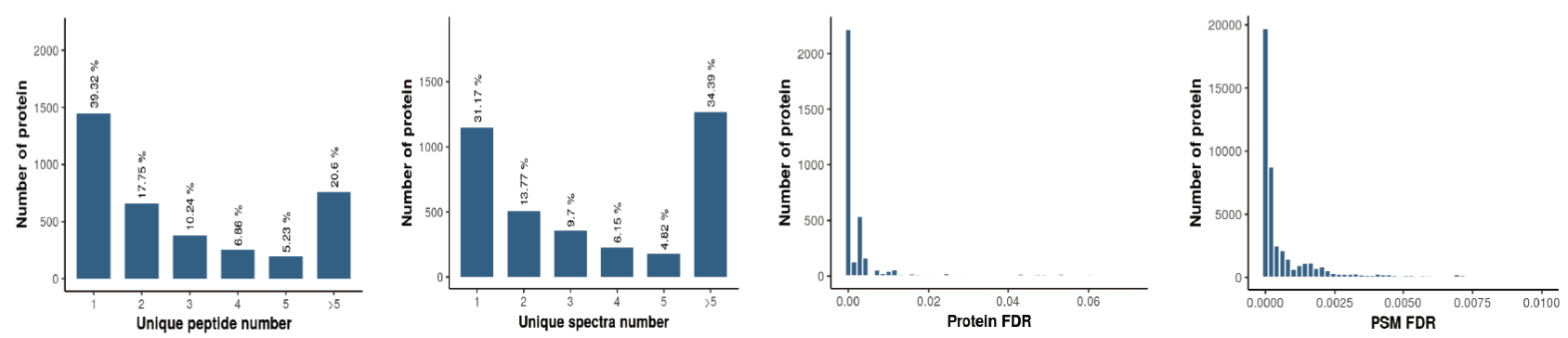

Figure S1 Quality control of the data identified by proteomics in rat bladder tissue. (a) Protein molecular weight distribution diagram, peptide charge distribution diagram and missed cleaveges distribution diagram. (b) Protein identification unique peptide number distribution diagram, protein identification unique spectra number distribution diagram, protein false discovery rate and peptide-spectrum match false discovery rate. 


\section{Appendix 2}

We display the top 20 proteins with increased or decreased expression and their fold change in the following table.

Table S1 The top 20 increased differentially expressed protein (DEPs) in ketamine treated rat urine samples compared to control group

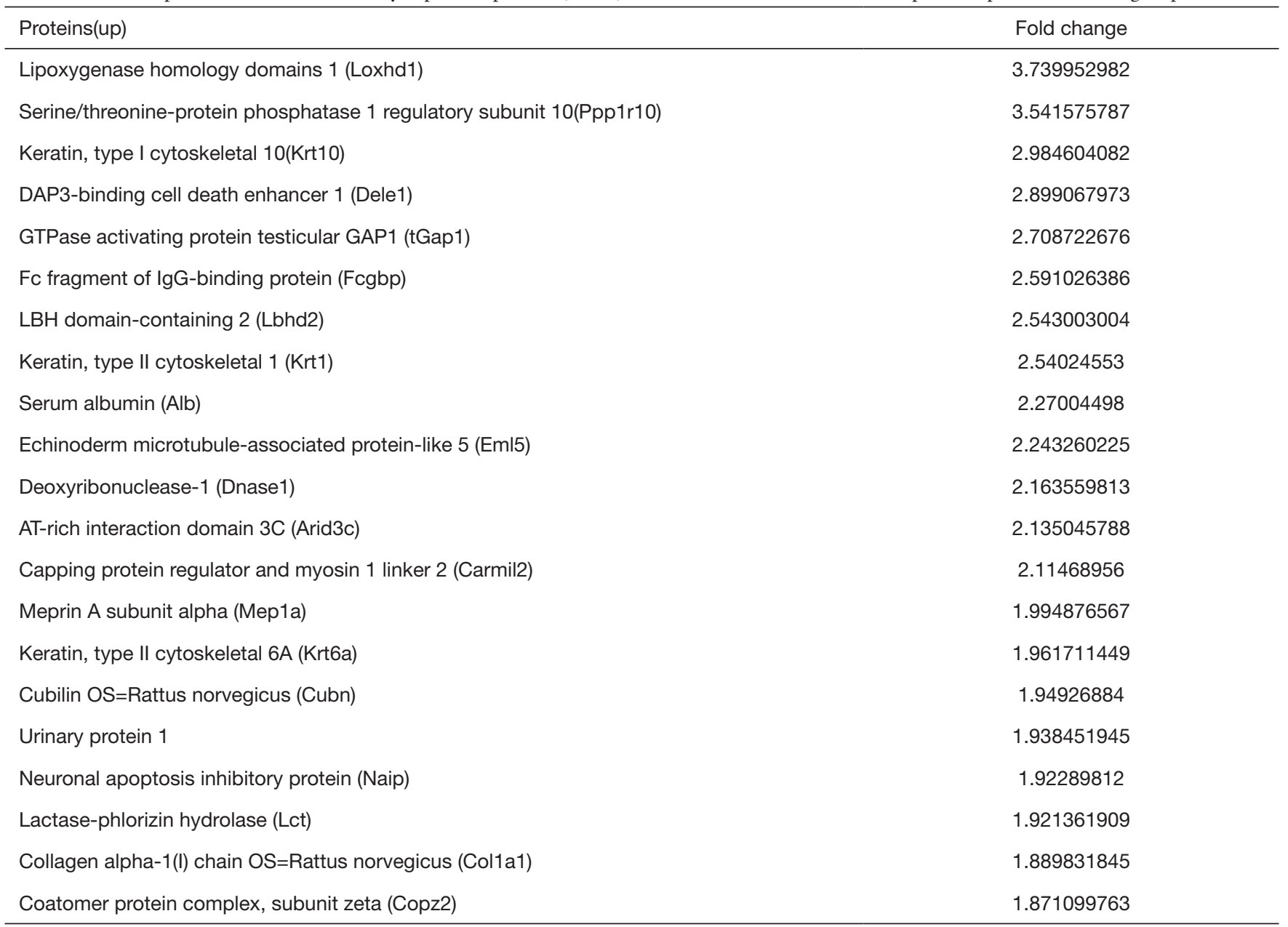


Table S2 The top 20 decreased differentially expressed protein (DEPs) in ketamine treated rat urine samples compared to control group

\begin{tabular}{|c|c|}
\hline Proteins (down) & Fold change \\
\hline Mast cell protease 2 (Mcpt2) & 0.391628906 \\
\hline Acyloxyacyl hydrolase (Aoah) & 0.475499379 \\
\hline Zero beta-globin (Fragment) & 0.494807389 \\
\hline Parvalbumin alpha (Pvalb) & 0.496477493 \\
\hline GIPC PDZ domain-containing family, member 3 (Gipc3) & 0.502031679 \\
\hline Aly/REF export factor (Alyref) & 0.529845212 \\
\hline Alpha globin (Hba-a3) & 0.535662499 \\
\hline Keratin, type I cytoskeletal 14 (Krt14) & 0.571095288 \\
\hline Four and a half LIM domains 3 (Fhl3) & 0.594009377 \\
\hline Voltage-dependent calcium channel subunit alpha-2/delta-3 (Cacna2d3) & 0.594103368 \\
\hline HECT domain E3 ubiquitin protein ligase 4 (Hectd4) & 0.599442607 \\
\hline Olfactory receptor OS=Rattus norvegicus (Olr174) & 0.602178973 \\
\hline Actin, beta-like 2 OS=Rattus norvegicus (Actbl2) & 0.60514843 \\
\hline Alpha-synuclein OS=Rattus norvegicus (Snca) & 0.611749025 \\
\hline
\end{tabular}




\section{Appendix 3}

We compared the DEPs and the COG database to obtain annotations for each protein, and macroscopically recognize which COG the identified protein belongs to. We also predicted the possible functions of these proteins and classified them through their functions. The functional classification with the largest number of proteins were general function prediction only, with 39 proteins involved; the second functional classification was posttranslational modification, protein turnover, chaperones (34 proteins), translation, ribosomal structure and biogenesis (29 proteins), signal transduction. mechanisms (19 proteins) (Figure $S 2 A)$.

After getting the EggNOG (evolutionary genealogy of genes: Non-supervised Orthologous Groups) annotation for each protein, we done EggNOG statistics on the protein to identify which EggNOG the protein belongs to. And the database mainly divided into five categories: cellular processes and signaling, energy production and conversion, information storage and processing, metabolism, poorly characterized. The results showed that DEPs involed most in cellular processes and signaling (618 proteins), accounting for $48 \%$ of all identified proteins, in cellular processes and in signaling, proteins were mostly involved in signal transduction mechanisms (167 proteins), followed by posttranslational modification, protein turnover, chaperones (137 proteins), intracellular trafficking, secretion, and vesicular transport (121 proteins), cytoskeleton (95 proteins) (Figure $S 2 B$ ).

Proteins can only perform their specific functions in specific subcellular locations. We obtained subcellular localization annotations for each protein and performed statistical analysis. The results showed that the number of proteins was highest in cytoplasm (254 proteins), followed by nucleus (86 proteins), extracellular (130 proteins) (Figure S2C). After annotating parallel statistical analysis of the transcription factors of each identified protein, the number of proteins involved in transcription cofactors was the highest (20 proteins), followed by zf-C2H2 (4 proteins), chromatin remodeling factors (3 proteins) (Figure $S 2 D$ ). 
A

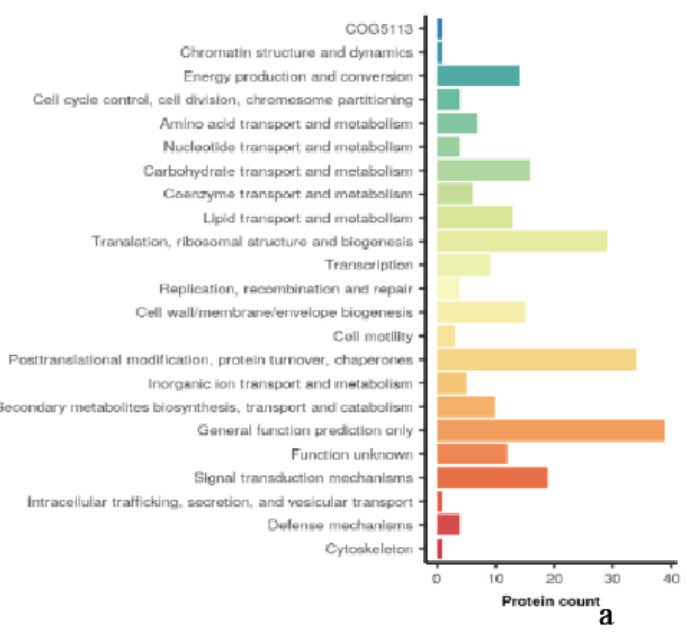

C

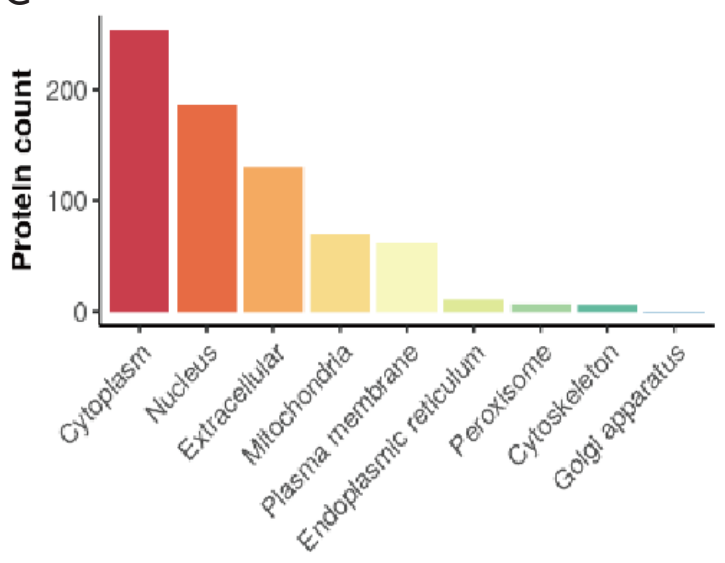

B

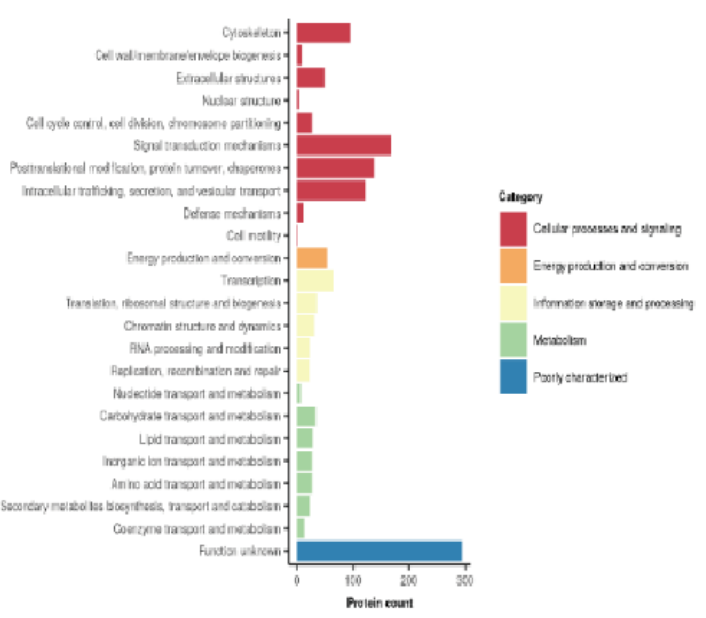

D

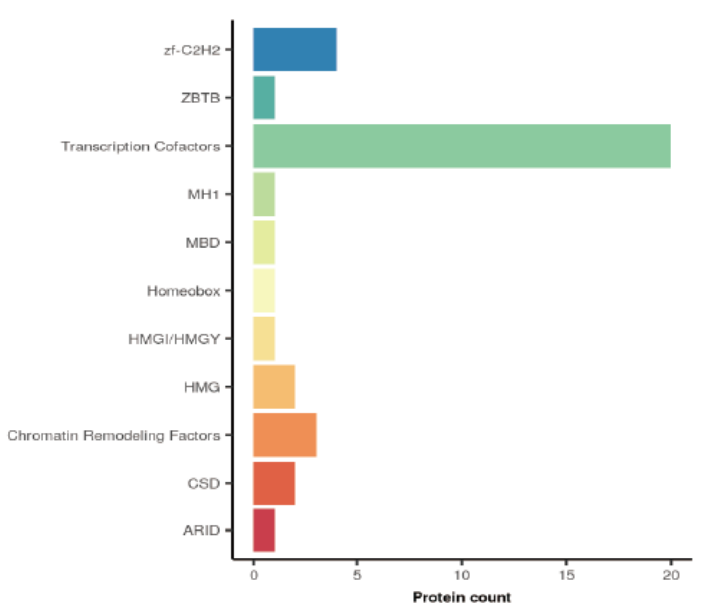

Figure S2 Functional annotation of DEPs. (a) Statistics of COG entries of DEPs. (b) Statistics of EggNOG entries of DEPs. (c) Statistics of subcellular localization entries of DEPs. (d) Statistics of transcription factors entries of DEPs. 


\section{Appendix 4}

In the platelet activation pathway, 64 proteins are annotated, 19 of which are differentially expressed, $\mathrm{P}=0.03212282$. For information about each protein and its expression, see Table $S 4$ and Figure $S 3$.

Table S3 KEGG annotation for platelet activation

\begin{tabular}{lcccccc}
\hline Pathway & $\begin{array}{c}\text { Diff Proteins with pathway } \\
\text { annotation (662) }\end{array}$ & $\begin{array}{c}\text { All Proteins with pathway } \\
\text { annotation (3394) }\end{array}$ & Pvalue & Qvalue & Pathway ID & Level 1 \\
\hline $\begin{array}{l}\text { Platelet } \\
\text { activation }\end{array}$ & $19(2.87 \%)$ & $64(1.89 \%)$ & 0.03212282 & 0.7091730262 & ko04611 & $\begin{array}{c}\text { Organismal } \\
\text { Systems }\end{array}$ \\
\hline
\end{tabular}

Table S4 All proteins differentially expressed in the platelet activation pathway

\begin{tabular}{ll}
\hline Pathway & Proteins \\
\hline Platelet & tr|D4ACS0|D4ACS0_RAT, tr|A0A0G2JU01|A0A0G2JU01_RAT,sp|Q63538|MK12_RAT, sp|P62836|RAP1A_RAT, \\
activation & sp|P02454|CO1A1_RAT, tr|Q9R1X8|Q9R1X8_RAT, sp|P10824|GNAl1_RAT, tr|Q45QM8|Q45QM8_RAT, sp|P14480|FIBB_RAT, \\
& tr|G3V852|G3V852_RAT, sp|P61589|RHOA_RAT, tr|A1L114|A1L114_RAT, tr|Q45QN0|Q45QN0_RAT, tr|M0R4J7|M0R4J7_ \\
& RAT, tr|D3ZRN3|D3ZRN3_RAT, tr|F1LS40|F1LS40_RAT, sp|Q76K24|ANR46_RAT, tr|D4ACC2|D4ACC2_RAT, \\
& tr|F1LQ3|F1LQT3_RAT \\
\hline
\end{tabular}




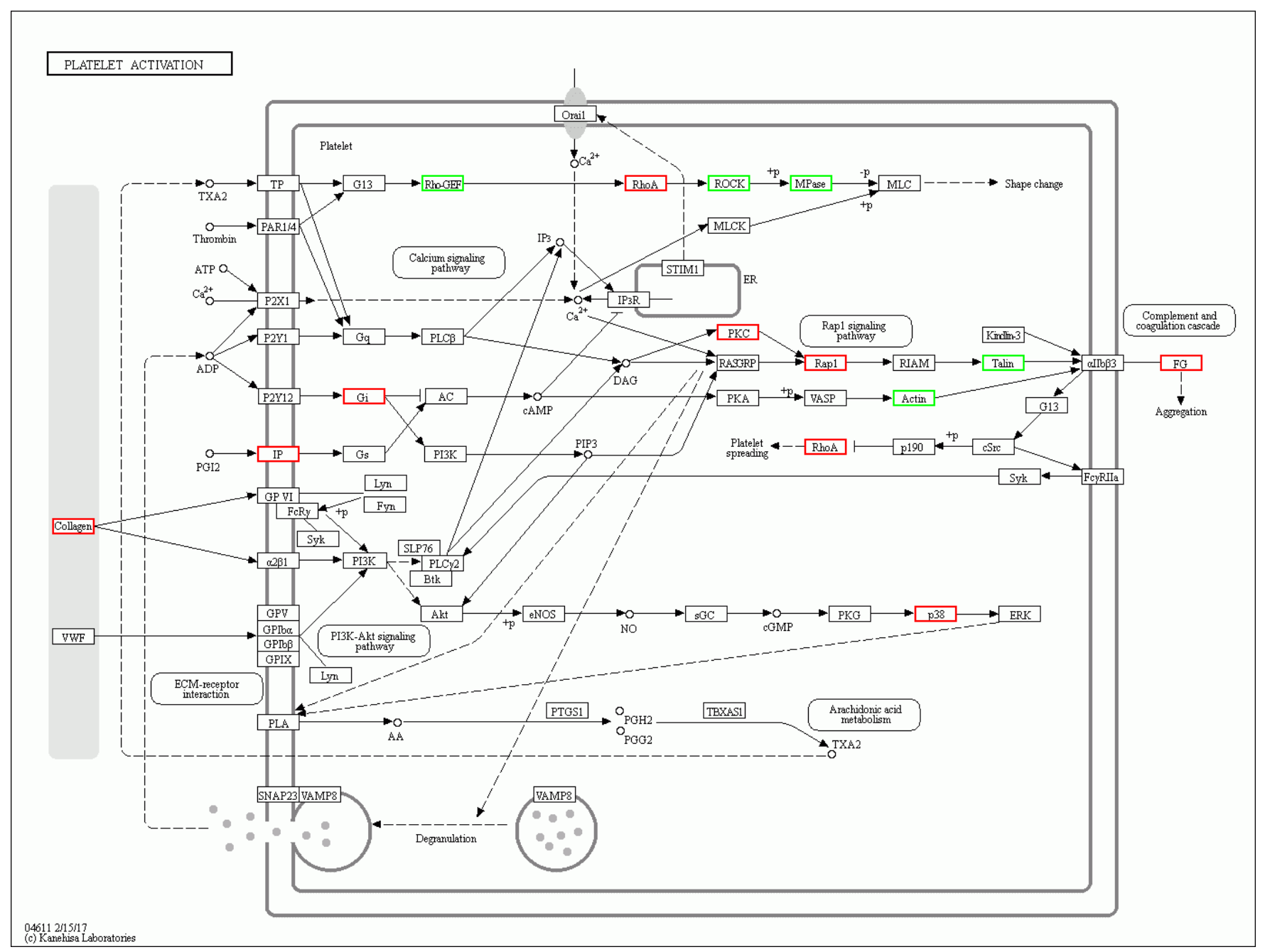

Figure S3 The KEGG pathway diagram of the platelet activation pathway. 